\title{
Longitudinal Modeling and Control of Tailed Flapping-Wings Micro Air Vehicles near Hovering
}

\author{
Tao Jiang $\left(\mathbb{D},{ }^{1,2,3}\right.$ Xiaosong Yang, ${ }^{4}$ Hongwei Wang, ${ }^{1,2}$ Wen Gai, ${ }^{4}$ and Long Cui ${ }^{1,2}$ \\ ${ }^{1}$ State Key Laboratory of Robotics, Shenyang Institute of Automation, Chinese Academy of Sciences, Shenyang 110016, China \\ ${ }^{2}$ Institutes for Robotics and Intelligent Manufacturing, Chinese Academy of Sciences, Shenyang 110016, China \\ ${ }^{3}$ University of Chinese Academy of Sciences, Beijing 100049, China \\ ${ }^{4}$ Facility Design and Instrumentation Institute, China Aerodynamics Research and Development Center, Mianyang 621000, China
}

Correspondence should be addressed to Tao Jiang; jiangtau@outlook.com and Long Cui; cuilong@sia.cn

Received 18 June 2019; Accepted 18 July 2019; Published 22 August 2019

Academic Editor: Yangmin Li

Copyright (c) 2019 Tao Jiang et al. This is an open access article distributed under the Creative Commons Attribution License, which permits unrestricted use, distribution, and reproduction in any medium, provided the original work is properly cited.

\begin{abstract}
Compared with the tailless flapping wing micro air vehicle (FMAV), the tailed FMAV has a simpler structure and is easier to control. However, although biplane FMAVs with tails have been used for flight control in practice for a long time, a theoretical model of the tailed FMAV has not previously been established. In this paper, we report modeling of the longitudinal dynamics of a tailed biplane FMAV using the Newton-Euler equations. In this study, the vehicle was trimmed and linearized near its hovering equilibrium, assuming small disturbances. Then the stability of the hovering FMAV was analyzed with a modal analysis method. A state feedback controller was synthesized to stabilize the disturbance. Finally, we investigated the flight control of the tailed biplane FMAV with different control signals. Our results show that the natural-motion mode determines the oscillation divergence characteristics of the tailed FMAV, a mode that can be suppressed with the state feedback controller by real-time modulation of the tail. The tail can also be used to achieve different flight modes with different control-signal functions. The tailed FMAV cruises in a line when the tail is controlled with a step function and spirals in an elliptical trajectory in the longitudinal plane when the tail is controlled by a sinusoidal function. Our longitudinal- dynamics model provides an analytical basis for further dynamic analyses of the tailed FMAV, as well as the corresponding controller synthesis. Moreover, the proposed attitude stabilization and flight control schemes for the vehicle near hovering provide a basis for developing practical uses of the tailed FMAV.
\end{abstract}

\section{Introduction}

The high-efficiency, maneuverability, and small size of the flapping-wing micro air vehicle (FMAV) make it potentially very valuable in military or civilian applications such as unmanned reconnaissance and search and rescue in confined spaces. An essential prerequisite for achieving these uses is stable flight and maneuverability. Furthermore, stable hovering of the aircraft and controlling its flight near hovering play a vital role in the collection of high-quality information. For this flight mode, birds and insects provide useful references.

Biological studies have shown that birds and insects control their wings differently. An insect cannot actively change the shape of its wing membrane when flapping its wings. Instead, the insect can only coordinate the multiple kinematic parameters of the wing root to control the wing motion and generate asymmetric aerodynamic forces, thereby achieving hovering and controlling flight attitude [13]. A bird modulates the morphology of its wings and the swing of its tail through muscle tissue to achieve attitude control. However, most birds cannot hover because of the small pitch angle of their wings during flight [4-6]. It can be concluded that there are two main distinctions between the bird's and the insect's flapping method: how many degrees of freedom of wing motion there are and whether there is a tail involved in flight control. In the past several years, numerous FMAVs emerged resembling insects or birds. These FMAVs have different characteristics as described below.

The "robotic fly" is a typical insect-like FMAV. It uses piezoelectric actuators to independently drive its two wings and generates a control torque through asymmetric wing flapping to adjust its flight attitude. However, due to load 
limitations, flights without a wire are not currently available [7]. Ramenzani proposed a bat-inspired FMAV that can fly without a guiding wire. It uses a linkage mechanism to imitate the front and rear arms of the bat and control the integrated wing-membrane structure to achieve flight modes such as cruise and glide [8]. Different from insects and birds, the bat's blended wing body with a flexible membrane leads to a strong fluid-structure interaction (FSI) phenomenon, which increases the difficulty of flight control. We have found no reports of successful hover function for the bat FMAV.

The "nano hummingbird" is an FMAV with hovering ability inspired by the hummingbird. It generates thrust and lift through its flapping wings and modulates the distribution of wing twist by changing the angle of the root spar to the leading edge, all of which generate roll and yaw torque to achieve attitude control [9]. Phan's "KUBeetle" has a flapping mechanism similar to that of the nanohummingbird and uses PD feedback to stabilize flight attitude [10]. Both FMAVs mentioned above used hummingbirds as bionic reference objects, but both abandoned the hummingbird's tail as a control mechanism. Studies have shown that hummingbirds actively adjust the flutter amplitude of the tail and the direction of the central axis of the fan angle in turbulence to increase its passive stability [11]. The tailless FMAV is not passively stable [12], and the wing motion must be actively fed back to achieve attitude stabilization, such as Karasek's tailless biplane-winged MAV did [9]. The real-time control method increases the complexity of the aircraft driving mechanism and limits its further miniaturization. Moreover, the complicated mechanism increases the weight as well as energy consumption, reducing flight duration. From a practical point of view, improving the endurance time and reducing the complexity of the flapping driving mechanism without jeopardizing the FMAV's flight stability or hovering ability are important research directions.

In this respect, an FMAV with a tail has an advantage. The delfly series of FMAVs is representative of this kind of vehicle [13]. The wings of this kind of FMAV can only produce aerodynamic forces in a fixed direction, which must be controlled by the deflection of the tail [14]. In fact, relying on the tail to provide both stability and a flight control surface is precisely its advantage, as this makes it possible to reduce the complexity of the control mechanism. Armanini modeled the aerodynamic interaction between the tail and the wake produced by the wings when an FMAV cruises at low speed [15]. By studying the influence of the clap process on the aerodynamics of the biplane FMAV and also the clap-and-fling aerodynamic mechanism, Jadhav concluded that there exists a continuous interaction between the tail and the wake of the forward wings during hovering [16]. Jadhav's findings indicate that the aerodynamic model of the tail proposed by Armanini should also apply to the biplane FMAV in the vicinity of its hovering state.

Research is still limited on hovering of the biplane FMAV as well as on its flight control during hovering. In order to fill this gap, we conducted research along the following lines. First, we modeled the longitudinal dynamics of the tailed
FMAV using the Newton-Euler equation. The flight equilibrium of the vehicle in hovering was adjusted with the tail deviation angle, and the longitudinal dynamics were linearized around the equilibrium point based on a small-disturbance assumption. We then analyzed the longitudinal stability of the tailed vehicle with a modal analysis method. The longitudinal response of the hovering vehicle when disturbed was also investigated.

Next, to stabilize the effects of disturbances on the FMAV, we designed a state feedback controller. Finally, we investigated flight control from hovering equilibrium with a tail. Our results show that the tailed biplane FMAV can maneuver in flight to achieve modes of flight such as hovering, cruising, and longitudinal spiraling. Achieving these flight modes is necessary for practical application of the FMAV, such as real-time exploration and field investigations. Therefore, our work expands the potential range of applications of the flapping wing micro aerial vehicle.

\section{Materials and Methods}

2.1. Simulation Model and Coordinate System. A tailed biplane FMAV is depicted in Figure 1. The vehicle consists of two pairs of upper and lower wings in an X configuration. The wings are driven by two sets of crank rocker mechanisms with a coreless brushless DC motor. The horizontal tail is located at the rear of the vehicle fuselage. Under the control of the electromagnetic rudder, a horizontal deviation angle can be generated. There exist a fixed inertial coordinate system $X_{e} Y_{e} Z_{e}$ and a body coordinate system $X_{b} Y_{b} Z_{b}$ with origins at the center of gravity (CoG) of the FMAV (marked with the red dot in Figure 1). Since the fuselage structure is bilaterally symmetrical and the left and right wings rotate as mirror opposites, the vehicle does not produce lateral motion. In this configuration, the aircraft only produces a longitudinal force $\mathrm{T}_{\mathrm{b}}\left(\mathrm{C}_{\mathrm{T}, \mathrm{w}}\right.$ in Figure 1) along the $X_{b}$ axis and a normal force $N_{b}$ $\left(C_{N, w}\right.$ in Figure 1$)$ along the $Z_{b}$ axis. When the FMAV wings flap, the leading-edge vortices (LEV) continually fall off the wing surface, shed from the trailing edge of the wing into the wake, which interacts with the tail to produce a tangential force $T_{t}\left(C_{T, t}\right.$ in Figure 1) and a normal force $N_{t}\left(C_{N, t}\right.$ in Figure 1).

\subsection{Aerodynamic Model and Dynamic Equation of Motion}

2.2.1. Quasistatic Aerodynamic Model. On condition that the practical flapping frequency of the wings is much higher than the natural frequency of the FMAV wing motion, the precondition of quasistatic assumptions satisfies. A timeaveraged aerodynamics coefficient can be used to model the flight dynamics, although the FMAV produces unsteady aerodynamics.

Engineering practice calls for first modeling the wing kinematics, based on which the instant aerodynamic forces and moments can be obtained by numerically solving the Navier-Stokes equation. The time-averaged aerodynamic forces and moments are then calculated. Finally, the 


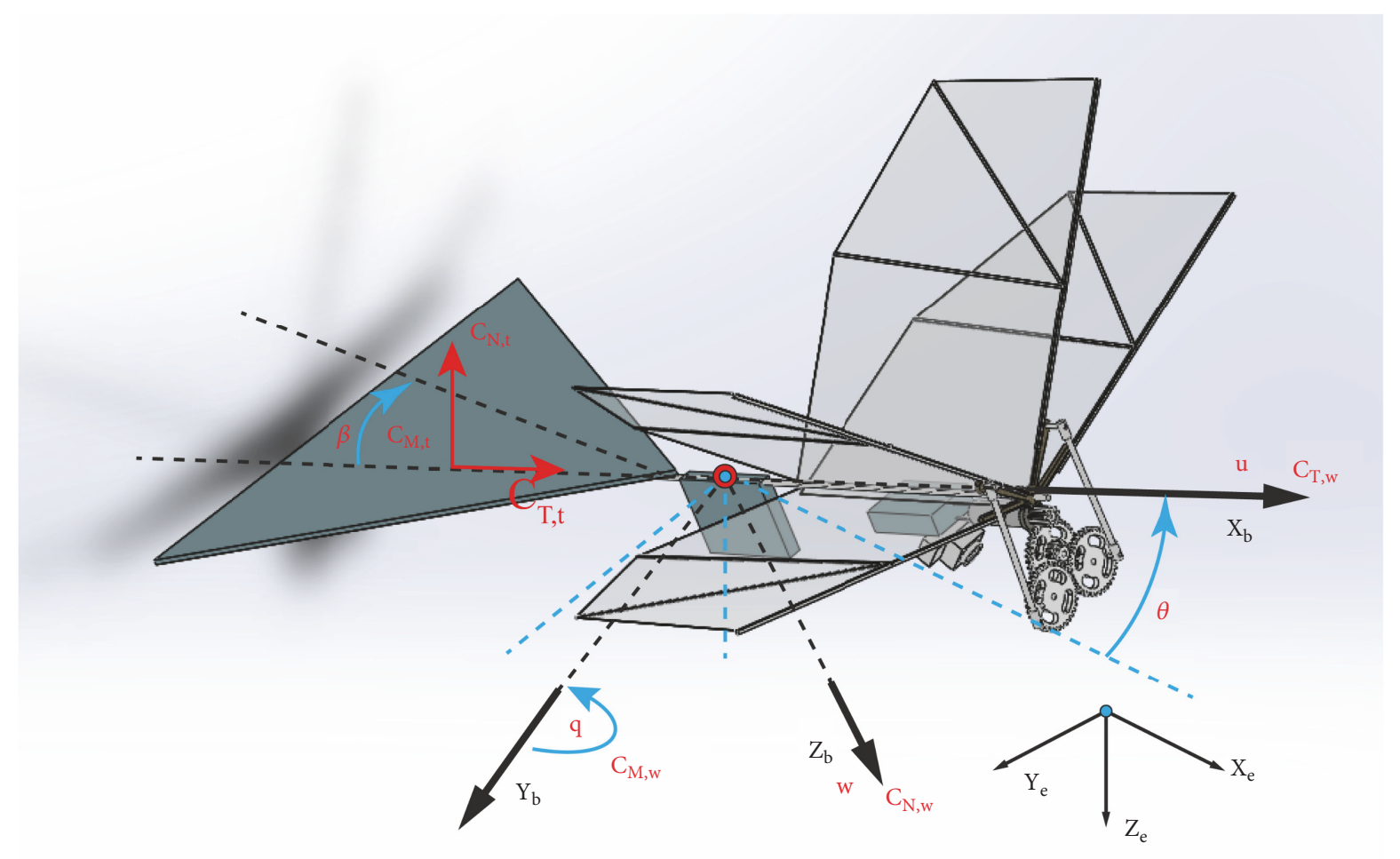

FIGURE 1: Diagram of the mechanism of tailed biplane FMAV and its parameter definitions.

aerodynamic coefficients of forces and moments, such as $C_{T}$, $C_{N}$, and $C_{M}$, are determined:

$$
\begin{gathered}
C_{T}=\frac{2 X}{\rho v^{2} S} \\
C_{N}=\frac{2 Z}{\rho v^{2} S} \\
C_{M}=\frac{2 M}{\rho v^{2} S}
\end{gathered}
$$

Here $X$ is the tangential aerodynamic force along the $X_{b}$ axis, $Z$ is the normal aerodynamic force along the $Z_{b}$ axis, $M$ is the aerodynamic pitching moment around the $Y_{b}$ axis, $\rho$ is the air density, $S$ is the wing area, and $v$ is the reference speed.

\subsubsection{Longitudinal Dynamics of the FMAV. The FMAV is} essentially a multibody dynamic system, which is more difficult to control than the fixed-wing aircraft. However, Caetano's research shows that the flapping wing can be modeled as a single rigid body, like a fixed-wing aircraft, without much error if the wing weight is less than $5 \%$ of the whole vehicle [17]. Under the single rigid-body assumption, the dynamics of an FMAV in hovering can be approximated in the body coordinate system $X_{b} Y_{b} Z_{b}$ with the Newton-Euler equation of motion. Due to the bilateral symmetry of the wings and its motion, the sideways thrust and torque cancel instantly. As a result, there is not sideslip or the lateral velocity.
Consequently, the longitudinal dynamic equations are obtained, as follows:

$$
\begin{aligned}
X-m g \sin \theta & =m(\dot{u}+q w) \\
Z+m g \cos \theta & =m(\dot{w}-q u) \\
M & =I_{y} \dot{q} \\
\dot{\theta} & =q
\end{aligned}
$$

Here $u$ and $w$ are, respectively, the forward flight velocity and the fluctuating velocity in the body coordinate system; $q$ and $\theta$ are, respectively, the pitch angular velocity and the pitch angle around the pitch axis $Y_{b}$. The direction of each state variable points in the positive direction of the coordinate system axis (as shown in Figure 1). The mass of the aircraft is $m=4.4 \times 10^{-3} \mathrm{~kg}, g=9.8 \mathrm{~m} / \mathrm{s}^{2}$ is the acceleration of gravity, and $I_{y}=1.73 \times 10^{-5} \mathrm{~kg} \cdot \mathrm{m}^{2}$ is the pitching moment of inertia of the vehicle. For convenience in the follow-up discussion, (2) are used to make (1) nondimensional, as follows:

$$
\begin{aligned}
C_{T}-m^{+} g^{+} \sin \theta & =m^{+}\left(\dot{u}^{+}+q^{+} w^{+}\right) \\
C_{N}+m^{+} g^{+} \cos \theta & =m^{+}\left(\dot{w}^{+}-q^{+} u^{+}\right) \\
C_{M} & =I_{y}^{+} \dot{q}^{+} \\
\dot{\theta} & =q^{+}
\end{aligned}
$$


Here $m^{+}=m / 0.5 \rho S c=45.4, g^{+}=g c / U^{2}=49.6$, and $I_{y}^{+}=I_{y} / 0.5 \rho S c^{3}=0.0278$ are the dimensionless quantities, $S$ is the wing area, $c$ is the chord length, and $\rho$ is air density. The tangential aerodynamic force $C_{T}$, the normal aerodynamic force $C_{N}$, and the pitching aerodynamic moment $C_{M}$ have two sources, i.e., wings and tail:

$$
\begin{gathered}
C_{T}=C_{T, w}+C_{T, t} \\
C_{N}=C_{N, w}+C_{N, t} \\
C_{M}=M_{M, w}+M_{M, t}
\end{gathered}
$$

Here the subscript $w$ represents the wing and $t$ represents the tail. We take the horizontal deviation angle of the tail as the control variable. Armanini obtained empirical formulas with experimental data for the tangential force $C_{T, t}$ and normal force $C_{N, t}$, which are produced from the interaction between the tail and the wake of the LEV near hovering [15]:

$$
\begin{aligned}
C_{T, t} & =C_{T 0, t} \cos ^{2} \beta+C_{T \pi / 2} \cdot \sin ^{2} \beta \\
C_{N, t} & =C_{N 0, t} \cdot \sin (2 \beta)
\end{aligned}
$$

Here $C_{T 0, t}=0.2, C_{T \pi / 2}=2.8$, and $C_{N 0, t}=1.6$ are constants obtained by fitting the experimental data, and $\beta$ is the horizontal deviation angle of the tail, as shown in Figure 1. Armanini's result was selected to model the aerodynamics of the tail since both of the FMAV prototypes share similar aerodynamic configurations and similar experimental conditions.

\subsection{Longitudinal Trim of the Vehicle}

2.3.1. Trim Condition at Equilibrium. The vehicle must be trimmed before linearizing the longitudinal nonlinear dynamics. Since the vehicle structure and the wing kinematics are laterally symmetric, the rolling angular velocity is zero. As a result, the lateral force and moment of the FMAV can both be considered balanced. The vehicle only needs trimming in the longitudinal direction using the horizontal deviation angle of the tail. When the FMAV is trimmed, both the resultant force and the moment at the CoG are zero. Considering the aerodynamic contribution from the tail (see (4)), we have

$$
\begin{aligned}
C_{T, w}+C_{T, t} & =0 \\
C_{N, w}+C_{N, t} & =-m^{+} g^{+} \\
C_{M, w}+C_{M, t} & =0
\end{aligned}
$$

Here $C_{M, t}$ is the aerodynamic pitching moment at the CoG produced by the tail, the formula for which is

$$
C_{M, t}=C_{T, t} \cdot l_{T, t}+C_{N, t} \cdot l_{N, t}
$$

where $l_{T, t}$ and $l_{N, t}$ are the distances between the tail and CoG in the tangential and normal directions. In this study, $l_{T, t} \approx 0$. In (7) and (5), $C_{M, t}$ can be simplified as follows:

$$
C_{M, t}=C_{N 0, t} \cdot \sin (2 \beta) \cdot l_{N, t}
$$

Here $l_{N, t}=63 \mathrm{~mm}$, which can be made nondimensional as $l_{N, t}^{+}=l_{N, t} / c=0.84$. Solving (6) and (8) yields, for the trimmed $\beta_{0}$,

$$
\beta_{0} \approx-0.037
$$

2.3.2. Linearization of the Longitudinal Motion Equation. When dealing with the problem of stability and attitude stabilization, the changes in the state variables are generally expected to be small within the neighborhood of equilibrium. Therefore, the longitudinal dynamic equation (3) can be approximated as a series of small deviation $\delta$ from the equilibrium point. The aerodynamic forces $C_{T}$ and $C_{N}$ and the aerodynamic moment $C_{M}$ are linear combinations of the state variables $\delta u^{+}, \delta w^{+}$, and $\delta q^{+}$and the control variable $\beta$, as well as the corresponding aerodynamic and control derivatives. Taking $C_{T}$ as an example, the linearization routine is presented as

$$
C_{T}=C_{T 0}+C_{T u} \delta u^{+}+C_{T w} \delta w^{+}+C_{T q} \delta q^{+}+C_{T \beta} \delta \beta
$$

where $C_{T u}, C_{T w}$, and $C_{T q}$ are the corresponding aerodynamic derivatives and $C_{T \beta}$ is the control derivative of the control variable $\beta$. One can linearize the longitudinal dynamics (3) by substituting (6) into (3) and expanding as in (10). The linearized longitudinal dynamics system is then

$$
\begin{aligned}
\delta \dot{u}= & \frac{C_{T u} \delta u^{+}}{m^{+}}+\frac{C_{T w} \delta w^{+}}{m^{+}}+\frac{C_{T q} \delta q^{+}}{m^{+}}+\frac{C_{T \beta} \delta \beta}{m^{+}} \\
& -g^{+} \delta \theta \\
\delta \dot{w}^{+}= & \frac{C_{N u} \delta u^{+}}{m^{+}}+\frac{C_{N w} \delta w^{+}}{m^{+}}+\frac{C_{N q} \delta q^{+}}{m^{+}}+\frac{C_{N \beta} \delta \beta}{m^{+}} \\
\delta \dot{q}^{+}= & \frac{C_{M u} \delta u^{+}}{I_{y}^{+}}+\frac{C_{M w} \delta w^{+}}{I_{y}^{+}}+\frac{C_{M q} \delta q^{+}}{I_{y}^{+}}+\frac{C_{M \beta} \delta \beta}{I_{y}^{+}} \\
\delta \dot{\theta}= & \delta q
\end{aligned}
$$

where $C_{N u}, C_{N w}, C_{N q}, C_{N \beta}, C_{M u}, C_{M w}, C_{M q}$, and $C_{M \beta}$ are aerodynamic derivatives and $C_{T \beta}, C_{N \beta}$, and $C_{M \beta}$ are control derivatives. For the convenience of subsequent analysis, one can convert into a state space form

$$
\left[\begin{array}{l}
\delta \dot{u} \\
\delta \dot{w} \\
\delta \dot{q} \\
\delta \dot{\theta}
\end{array}\right]=A\left[\begin{array}{l}
\delta u \\
\delta w \\
\delta q \\
\delta \theta
\end{array}\right]+B \cdot \beta
$$


TABLE 1: Nondimensionalized aerodynamic derivatives of FMAV near hovering.

\begin{tabular}{|c|c|c|c|c|c|c|c|c|}
\hline$C_{T u}$ & $C_{N u}$ & $C_{M u}$ & $C_{T w}$ & $C_{N w}$ & $C_{M w}$ & $C_{T q}$ & $C_{N q}$ & $C_{M q}$ \\
\hline-0.99 & -0.12 & 1.97 & -0.05 & -1.14 & 0.21 & -1.07 & -0.08 & -0.69 \\
\hline
\end{tabular}

TABLE 2: Eigenvalues of the system matrix.

\begin{tabular}{lcc}
\hline Mode $1: \lambda_{1,2}$ & Mode 2: $\lambda_{3}$ & Mode 3: $\lambda_{4}$ \\
\hline $2.057 \pm 10.8 i$ & -28.9 & -0.025 \\
\hline
\end{tabular}

where $A$ and $B$ are, respectively, the system matrix and control matrix:

$$
\begin{aligned}
& A=\left[\begin{array}{cccc}
\frac{C_{T u}}{m^{+}} & \frac{C_{T w}}{m^{+}} & \frac{C_{T q}}{m^{+}} & -g^{+} \\
\frac{C_{N u}}{m^{+}} & \frac{C_{N w}}{m^{+}} & \frac{C_{N q}}{m^{+}} & 0 \\
\frac{C_{M u}}{I_{y}^{+}} & \frac{C_{M w}}{I_{y}^{+}} & \frac{C_{M q}}{I_{y}^{+}} & 0 \\
0 & 0 & 1 & 0
\end{array}\right], \\
& B=\left[\begin{array}{c}
\frac{C_{T \beta}}{m^{+}} \\
\frac{C_{N \beta}}{m^{+}} \\
\frac{C_{M \beta}}{I_{y}^{+}} \\
0
\end{array}\right]
\end{aligned}
$$

The resultant linearized equations of longitudinal dynamics at the equilibrium are (12) and (13). Karasek compared the difference in the aerodynamic derivatives among several studies and concluded that the differences were due to different approximations and simplifications used [18]. Sun used methods of computational fluid dynamics (CFD) to analyze the aerodynamic forces of an insect, whose wings move with two degrees of freedom. Since most of the major unsteady aerodynamic mechanisms known for flapping wings were accounted for in Sun's study, the aerodynamic derivatives that were obtained are more realistic [19]. Sun further analyzed the aerodynamic derivatives of wings with different geometries [12], such as hoverfly, drone fly, crane fly, and hawk moths, of which the hawk moth's wing is the bionic prototype for our FMAV. As a result, aerodynamic derivatives of hawk moths were used for our research; these are summarized in Table 1 [12]. We analyze the longitudinal dynamics of the tailed FMAV based on these parameters.

\section{Results and Discussion}

3.1. Stability Analysis of Open Loop. The open-loop stability of a tailed FMAV was analyzed with eigenvalues and eigenvectors. According to (5) and (8), the system control matrix is

$$
B=\left[\begin{array}{c}
-0.0042 \\
0.07 \\
96.4 \\
0
\end{array}\right]
$$

To perform a stability analysis on the longitudinal dynamics, the system control variable can be set to zero. Substituting the aerodynamic derivatives in Table 1 into (13) yields the eigenvalues are shown in Table 2.

The modes shown in Table 2 for the longitudinal dynamic equation of the tailed biplane FMAV are an oscillating divergence motion mode (Mode 1), a fast convergence motion mode (Mode 2), and a slow convergence motion mode (Mode 3 ). The slow convergence mode (Mode 3 ) is mainly related to the fluctuating motion of the vehicle, while the oscillation divergence mode (Mode 1) and the fast convergence mode (Mode 2) are mainly related to the forward flight and to the pitch motion [19]. This means that the longitudinal channel of the FMAV is unstable due to the oscillating divergence mode (Mode 1 in Table 2).

To further investigate the effect of the oscillating divergence mode (Mode 1), we simulated the hovering tailed FMAV in an open-loop when disturbed. The initial values of $\delta u^{+}, \delta w^{+}$, and $\delta q^{+}$are all set to 0.01 for the initial state to simulate a disturbance to the hovering FMAV. The system state response is shown in Figure 2.

It can be inferred from this figure that even though a perturbation may be exerted on different kinematic state variables, the transient response settles into a similar behavior after several periods. Whereas $\delta u^{+}, \delta q^{+}$, and $\delta \theta^{+}$all oscillate and diverge, $\delta w^{+}$basically exhibits a stable convergence over time. Also to be noted is that the ratio of magnitudes of the state variables is constant for each simulated disturbance. For example, $\left|\delta q^{+}\right| /\left|\delta u^{+}\right| \approx 3$.

The similar behavior of state responses to different sources of disturbance can be explained by the natural-motion mode of the longitudinal dynamics of the tailed FMAV. From the perspective of vibration mechanics, the eigenvectors of the oscillating divergence mode of motion (Mode 1 in Table 2) determine the magnitudes and phases of the quantities measuring the disturbance, i.e., $\delta u^{+}, \delta w^{+}, \delta q^{+}$, and $\delta \theta^{+}$, relative to each other. These properties can be displayed more clearly by expressing the eigenvector in polar form. Table 3 shows the eigenvectors of the three natural-motion modes of the system matrix 


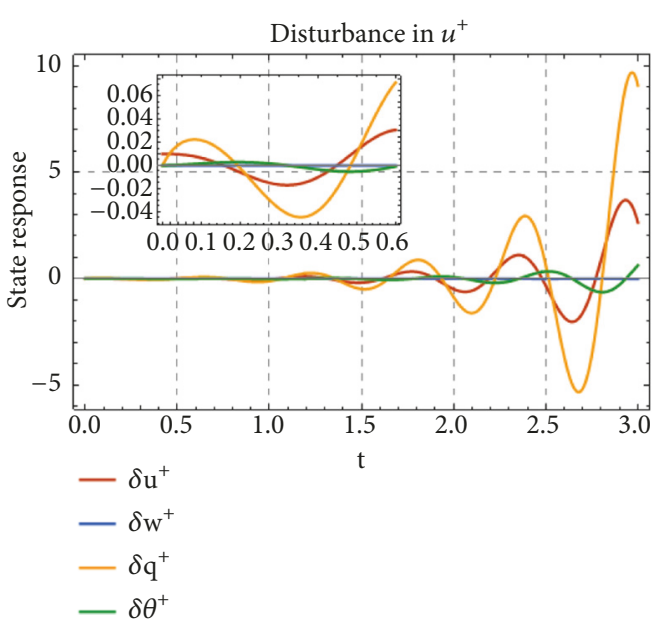

(a)

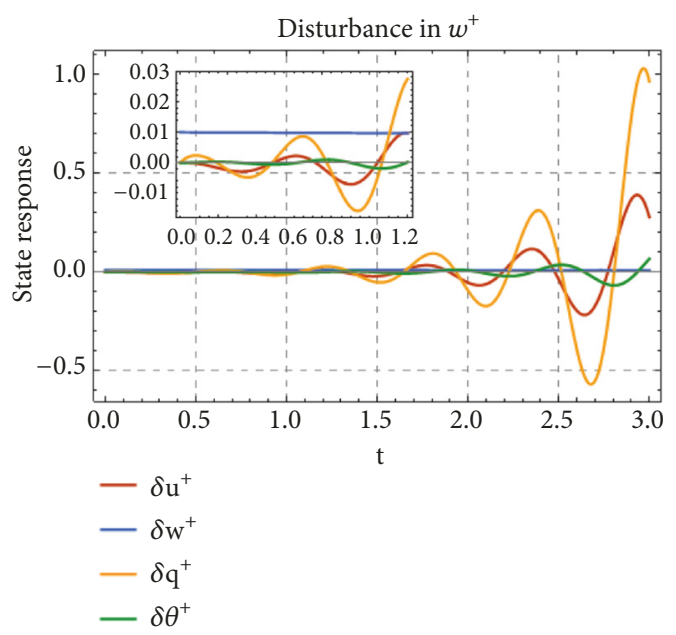

(b)

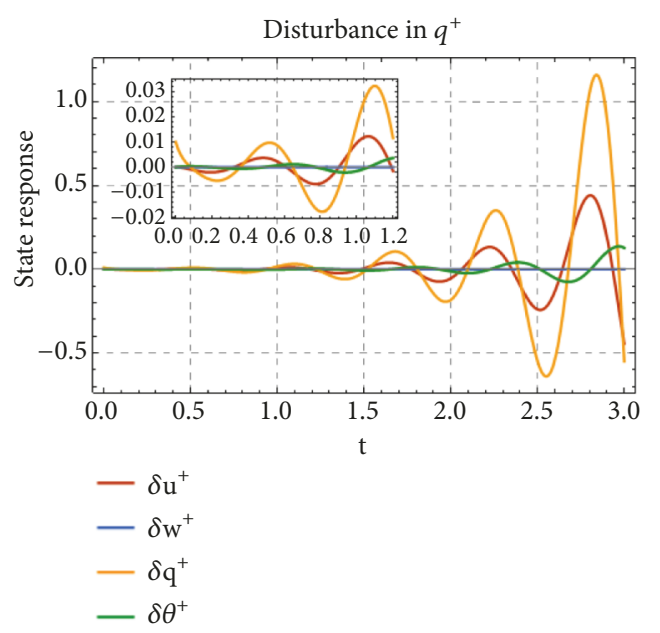

(c)

FIGURE 2: State response for disturbance of the open-loop system.

$A$ in Cartesian-coordinate form, and Table 4 shows the eigenvector of the oscillating divergence motion mode (Mode 1 in Table 2 or Table 3 ) in polar form.

These disturbances are given as initial angular velocity in $\delta u^{+}, \delta w^{+}$, and $\delta q^{+}$, and they can be viewed as a response with free damping to zero input. When the forward flight velocity $\delta u^{+}$, the fluctuating velocity $\delta w^{+}$, and the pitch angular velocity $\delta q^{+}$are respectively disturbed, the oscillating divergence mode in the longitudinal dynamics is excited, resulting in system instability.

As mentioned above, the magnitude and phase of the eigenvector components determine the magnitude and phase of the disturbance quantities, i.e., $\delta u^{+}, \delta w^{+}, \delta q^{+}$, and $\delta \theta^{+}$, relative to each other. For example, we can use $\delta q^{+}$and $\delta u^{+}$to illustrate the relationship in the magnitude of state responses in Figure 2.

As shown in Table $4,\left|\delta q^{+}\right| /\left|\delta u^{+}\right|=9.2 \times 10^{-1} / 3.77 \times$ $10^{-1} \approx 2.4$, which, for our purposes, can be rounded to 3 . In each different disturbing situation, as depicted in Figure 2, the ratio of the magnitudes of $\delta q^{+}$and $\delta u^{+}$is always about 3. Furthermore, the magnitude relationship between the eigenvector components in Table 4 implies that whenever the FMAV is disturbed, only $\delta \theta^{+}, \delta q^{+}$, and $\delta u^{+}$oscillate and then diverge, while the amplitude of $\delta w^{+}$changes very little. By analyzing the magnitude of eigenvector components of the unstable-motion mode, the critical state variables affecting system stability can be identified.

On the other hand, the phase difference between state variables excited by different disturbing sources varies. Consider the $\delta w^{+}$disturbance (Figure 2(b)) and the $\delta q^{+}$disturbance (Figure 2(c)) as examples. When $\delta w^{+}$is disturbed, $\delta q^{+}=0$ at $t=2.52$, while when $\delta q^{+}$is disturbed, $\delta q^{+}=0$ at $t=2.38$. There exists a time difference of $0.15 \mathrm{~s}$ between these two disturbing situations according to Figures 2(b) and 2(c). This time difference is caused by the different phase angles of $\delta q^{+}$and $\delta w^{+}$, which are also the eigenvector components in Table 4 . Due to the phase difference $\angle \delta q^{+}-\angle \delta w^{+}=$ $2.03 \mathrm{rad}$, the response of $\delta q^{+}$excited by a $\delta w^{+}$disturbance (Figure $2(\mathrm{~b}))$ lags by $(2.03 \mathrm{rad}) /(10.8 \mathrm{rad} / \mathrm{s})=0.18 s \approx 0.15 \mathrm{~s}$ behind the response of $\delta q^{+}$excited by a $\delta q^{+}$disturbance. Note 
TABLE 3: Eigenvectors of system matrix.

\begin{tabular}{lccc}
\hline & Mode 1 & Mode 2 & Mode 3 \\
\hline$\delta u^{+}$ & $0.349 \pm 0.141 i$ & 0.0582 & -0.106 \\
$\delta w^{+}$ & $-0.0001 \pm 0.0002 i$ & -0.0001 & 0.994 \\
$\delta q^{+}$ & 0.922 & -0.998 & 0 \\
$\delta \theta^{+}$ & $0.0156 \pm 0.0822 i$ & 0.0345 & 0 \\
\hline
\end{tabular}

TABLE 4: Amplitude and phase in polar form of components of eigenvector of Mode 1 in Table 3.

\begin{tabular}{|c|c|c|c|c|}
\hline Mode 1 & $\delta u^{+}$ & $\delta w^{+}$ & $\delta q^{+}$ & $\delta \theta^{+}$ \\
\hline \multirow{2}{*}{ Unstable oscillatory } & $3.77 \times 10^{-1}$ & $2.2 \times 10^{-4}$ & $9.2 \times 10^{-1}$ & $8.3 \times 10^{-2}$ \\
\hline & $0.38 \mathrm{rad}$ & $2.03 \mathrm{rad}$ & $0 \mathrm{rad}$ & $1.38 \mathrm{rad}$ \\
\hline
\end{tabular}

that $10.8 \mathrm{rad} / \mathrm{s}$ is the imaginary part of the eigenvalue of the oscillation mode (Mode 1 in Table 2), and it represents the oscillation angular frequency. The phase difference between the various state variables, determined by the eigenvector component, can help one design a phase lead correction or lag correction to improve the phase stability margin of the system.

It should be pointed out that although the aerodynamic derivatives used in the current study are the same as those of the hawk moth in Sun's research [12], the FMAV in our research diverges faster in its longitudinal dynamics under a disturbance. The difference may be because the dimensionless mass and pitching moment of inertia of the FMAV in this study exceeds that of the hawk moth, resulting in different eigenvalues (system poles) from those of the hawk moth. These findings suggest that the stability of the FMAV relates not only to its aerodynamic derivatives but also to the mass and load distribution (moment of inertia) of the vehicle, which suggests that a lightweight FMAV with compact design not only helps to reduce energy consumption but also contributes to the dynamic stability of the FMAV.

\subsection{Pitch Stabilization with State Feedback. Since the FMAV} is unstable in the longitudinal channel, the hovering flapping vehicle will show divergent motion soon after being disturbed. Thus the vehicle needs to be stabilized with real-time feedback control to achieve stable hovering. Wu and Sun decoupled the longitudinal dynamics into independent dynamic equations via motion modal analysis, with each system state being controlled by the flapping angle amplitude and the rotation angle amplitude above and below the horizontal plane [20], which realizes the longitudinal attitude stabilization of the bumblebee. They treated the longitudinal motion of the insect as a multi-input-multi-output control system, which reflects the typical control routine of the tailless flapping wings. This control method requires more control freedoms as well as a more complicated driving mechanism, which limits the further miniaturization of flapping-wing micro air vehicles.

A simple control method leads to a simple driving mechanism. We chose the horizontal deviation angle of the tail as the control variable to stabilize the longitudinal disturbance, which is standard for a single-input control system. In this case, a state feedback control scheme is frequently used. One can first determine the controllability of the system. The system controllability matrix is

$$
S=\left[\begin{array}{llll}
B & A B & A^{2} B & A^{3} B
\end{array}\right]
$$

Substituting from (13) and (14) into (15) and considering the aerodynamic derivatives in Table 1, one sees that

$$
\operatorname{rank}(S)=4=n
$$

meaning that $n=4$ is the order of the system matrix A. According to (16), the system is controllable. The system state can be fed back into the control variable $\beta$, as shown in Figure 3. Let the state feedback gain matrix be

$$
K=\left(k_{u}, k_{w}, k_{q}, k_{\theta}\right)
$$

The bumblebee can stabilize its attitude when disturbed and restores its initial stable hovering status within 6-10 wing flapping cycles [21]. Inspired by this observation, we chose the bumblebee's stabilizing time as the performance index to configure the closed-loop poles of the feedback control system. After several trials, a set of poles were obtained as $p_{1,2}=-6 \pm 0.1 i, p_{3,4}=-1 \pm 0.1 i$, and the state feedback gain matrix becomes

$$
K=(0.72,0.21,-0.11,0.62)
$$

For comparing the improvement of stability with the open-loop FMAV in Figure 2, we simulated the state response under disturbance of $\delta u_{0}=0.1, \delta w_{0}=0.1$, and $\delta q_{0}=0.1$. The reason to take 0.1 as the upper limit of disturbance is that only when the disturbance is less than 0.1 do the longitudinal motion equations (12) and (13) satisfy the assumption of small disturbance [20]. The state response of the closed-loop system is shown in Figure 4.

This figure shows that a tail-based feedback control scheme can suppress the disturbance to a hovering FMAV in the longitudinal channel quickly and smoothly, unlike for the open-loop system in Figure 2. Moreover, the vehicle can recover its initial attitude within 6-10 flapping cycles, even though the disturbance is amplified tenfold, which is consistent with observed insect behavior [22]. Unlike a tailless insect, which can coordinate its multiple wing 


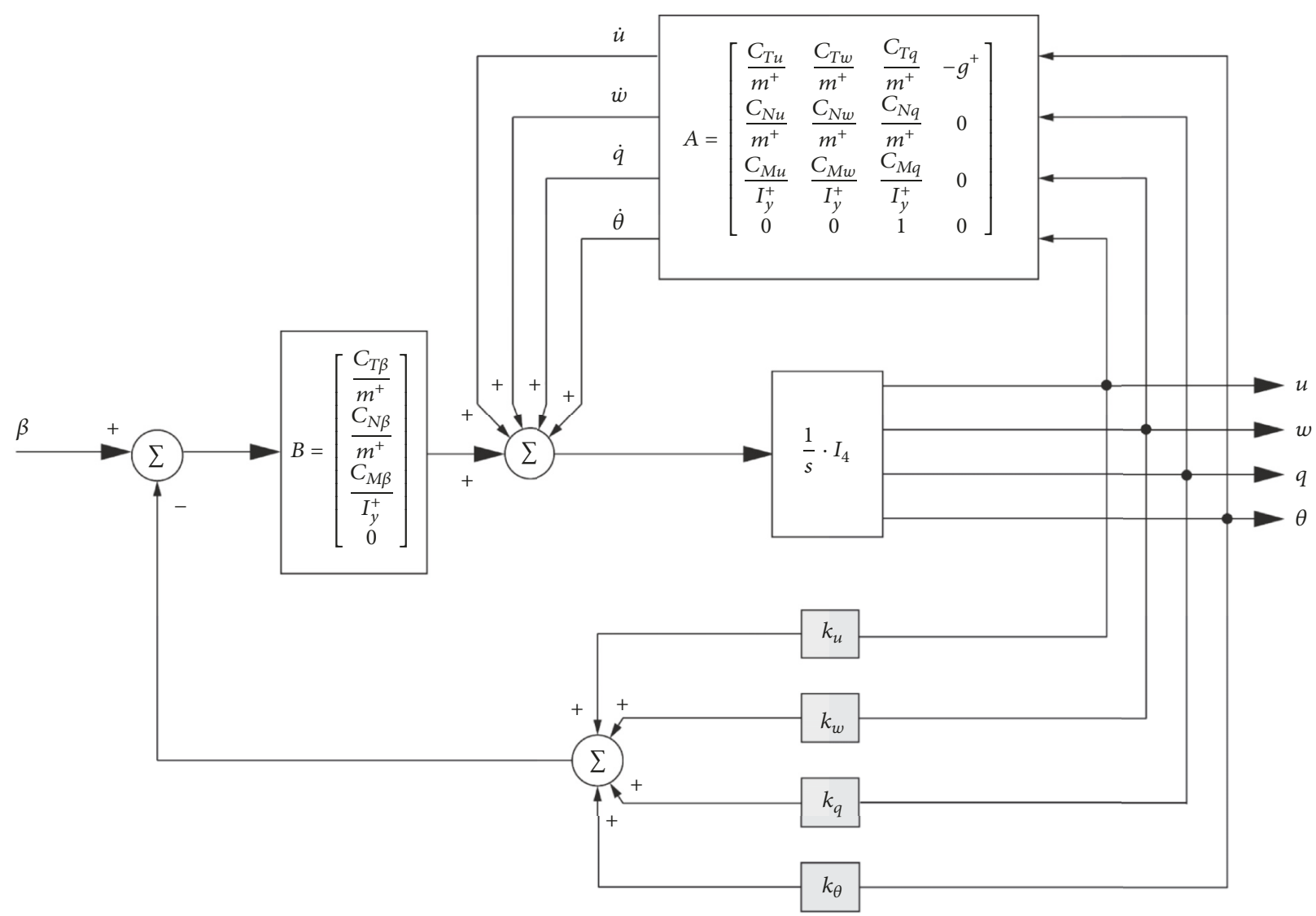

FIGURE 3: Scheme of state feedback control system.

kinematic parameters to achieve attitude stabilization [20], the tailed FMAV can achieve the same attitude stabilization just with the tail. Since there is only one control variable-the horizontal deviation angle $\beta$-the driving mechanism of the FMAV can be implemented with a compact and lightweight body frame.

Note that both the linearized longitudinal dynamics model and the consequent attitude stabilization controller are based on the assumption of small disturbances. Consequently, the result is not suitable for a large disturbance situation such as might be caused by gusts. Besides, for the disturbance to fluctuating velocity $\left(\delta w_{0}^{+}=0.1\right.$ in Figure $\left.3(\mathrm{~b})\right)$, the maximum of $\delta u^{+}$is 0.6 , which exceeds the upper limit of the small-disturbance assumption for linearization. To satisfy the linearization criterion of $\delta u_{\max }^{+}<0.1$, we should limit the maximum disturbance to the fluctuating velocity as $\delta w_{0, \max }^{+}=0.016$ (Figure $3(\mathrm{~b})$ ). The next step is to design a nonlinear controller to achieve attitude stabilization under the influence of large disturbances.

3.3. Flight Control from Hovering Equilibrium. Flight control near hovering is essential for unmanned reconnaissance, such as photographing and collecting high-quality information in other ways. The flight control for an FMAV mainly involves adjusting the velocity vector in the steady state. When pigeons cruise at low speed, they produce rhythmic movements at the tail, which can effectively reduce the vibration of the fuselage and improve flight stability [23]. Also, in turbulent flow, the hummingbird actively adjusts the flapping amplitude of the tail and the direction of the central axis of the sector angle to increase its passive stability [11]. These biological studies have shown that birds control their tails to assist in flight. Tail motion in different patterns may function in a similar way for the tailed FMAV. In this section, we report a study of the flight control of a tailed biplane FMAV with typical control signals such as step and sinusoid.

3.3.1. Tail Controlled with a Step Function. When the tail is controlled with a step function, the system state responds as shown in Figure 5, and the displacements of the relevant system state variables are as shown in Figure 6. The step function is

$$
\beta(t)= \begin{cases}\beta_{m}, & t \geq 0 \\ 0, & t<0\end{cases}
$$

where $\beta_{m}$ is the amplitude. Without loss of generality, we can temporarily set $\beta_{m}=0.005$.

As can be seen in Figure 5, if a step function controls the tail, the pitch angular velocity is $\delta q^{+}=0$ when the flight reaches the steady state, and the pitch angle displacement $\delta \theta^{+}$is almost zero. At the same time, both the forward flight 


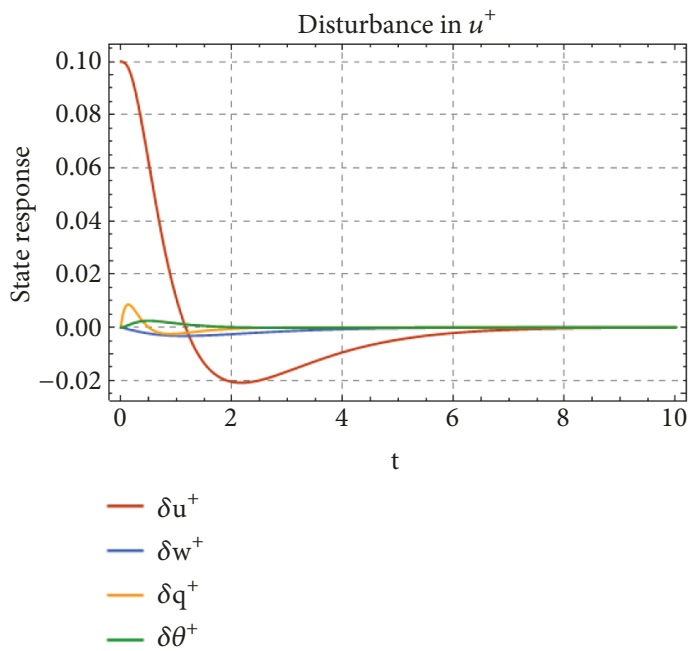

(a)

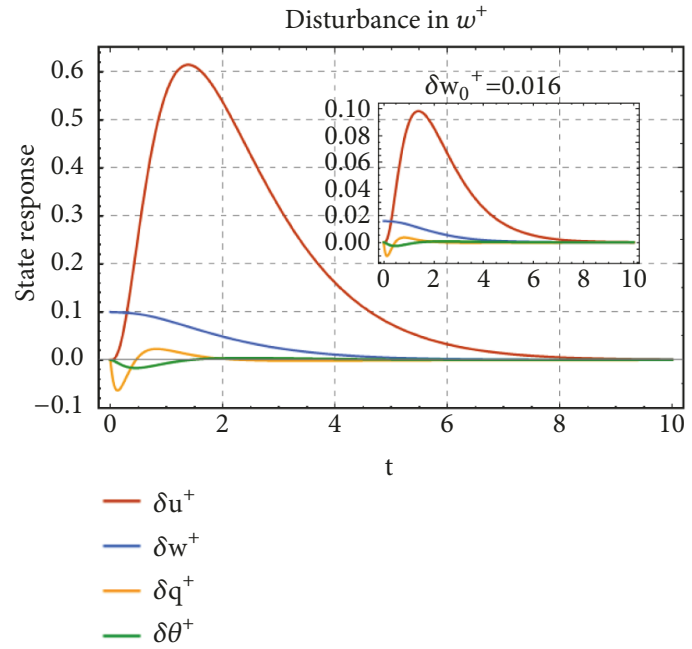

(b)

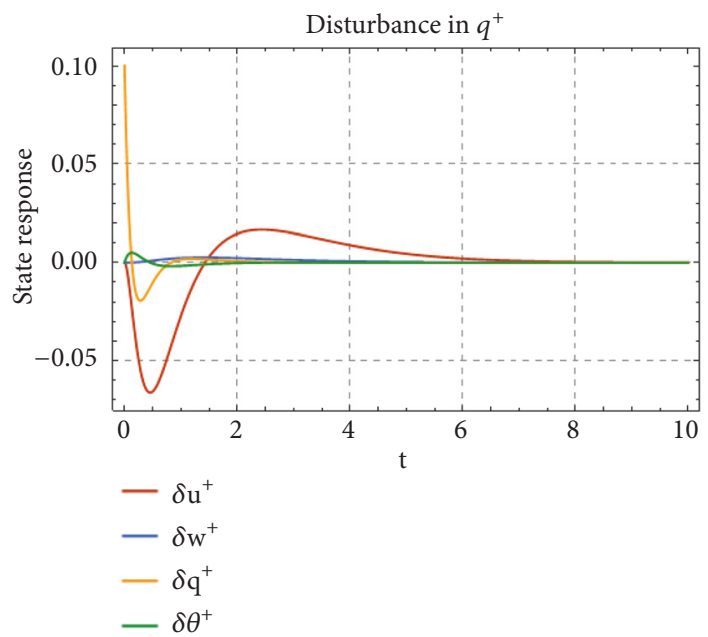

(c)

FIGURE 4: State response under disturbance of the state feedback control system.

velocity $\delta u^{+}$and the fluctuating velocity $\delta w^{+}$converge to constant values. In this case, the vehicle cruises in a line, as Figure 6 shows, which indicates that the FMAV moves both upwards and forwards. We further analyzed the general steady-state response of the system when the tail is controlled by a step function. First, the transfer function $c(s)$ of the system output is

$$
\begin{aligned}
& c(s)=\beta(s) \cdot \phi(s) \\
& \beta(s)=\int_{0}^{\infty} \beta(t) e^{-s t} d t \\
& \phi(s)=C(S \cdot I-A) B
\end{aligned}
$$

where $\phi(s)$ is the system's closed-loop transfer function, $\beta(s)$ is the Laplace transform of the step function, $c(s)$ is the transfer function of the system's output, $A$ and $B$ are the system matrix and the control matrix, respectively, as shown in (13), and $C$ is the system output matrix (here it is a 4 th order unit matrix). According to the Laplacian final value theorem (FVT), when the system reaches a steady state, its output is

$$
\lim _{t \longrightarrow \infty} c(t)=\lim _{s \longrightarrow 0} s \cdot c(s)
$$

Substituting the aerodynamic derivatives in Table 1 and the control derivative into (14), one finds the steady output of the system to be

$$
\begin{aligned}
& \lim _{t \rightarrow \infty} \delta u^{+}(t)=-1.68 \beta_{m} \\
& \lim _{t \rightarrow \infty} \delta w^{+}(t)=2.97 \beta_{m} \\
& \lim _{t \rightarrow \infty} \delta q^{+}(t)=0 \\
& \lim _{t \rightarrow \infty} \delta \theta^{+}(t)=0.00058 \beta_{m}
\end{aligned}
$$

According to (22) the pitch angular velocity is always $\delta q^{+}=0$ when the FMAV reaches steady state, and the 


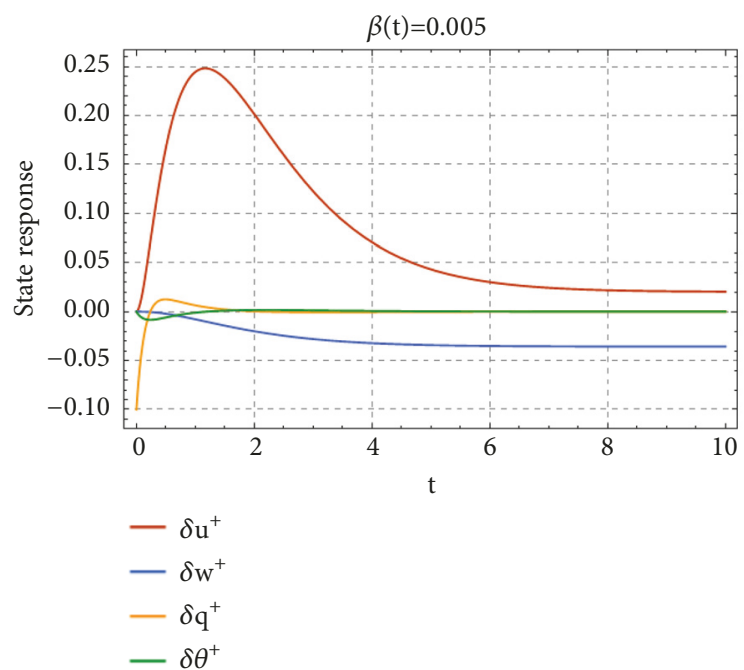

Figure 5: State response of an FMAV with its tail controlled by a step function.

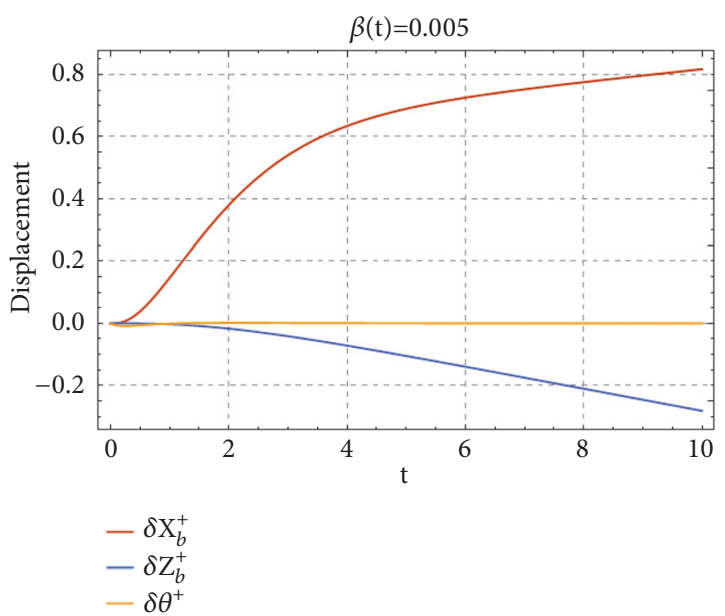

FIGURE 6: Displacement of an FMAV with its tail controlled by a step function.

pitch angle $\delta \theta^{+}$deviates from the trimmed pitch angle by $0.00058 \beta_{m}$, which is negligible, as indicated in Figure 5. The forward flight velocity $\delta u^{+}$and fluctuating velocity $\delta w^{+}$are proportional to $\beta_{m}$, and $u^{+} / w^{+} \approx-0.57$ is always constant, which indicates that the FMAV cruises at an upward angle if its tail is controlled by a step function with an amplitude of $\beta_{m}$. Meanwhile, its longitudinal attitude remains stable $\left(\delta q^{+}=0\right)$, and $\delta \theta^{+}$, the change in pitch angle, is negligible. The flight trajectory is a straight line that will tilt up or down, depending on the value of $\beta_{m}$.

3.3.2. Tail Controlled with a Sinusoidal Function. If the horizontal deviation angle of the tail is a sinusoidal function, we may, without loss of generality, assume that the horizontal tail oscillates at the same frequency as the flapping wings. The sinusoidal function controlling the tail is given by

$$
\beta(t)=\beta_{m} \cdot \sin t, \quad t \geq 0
$$

The corresponding phase diagram of the system state variables in the 2-dimensional phase plane is shown in Figures $7(a)-7(c)$. These figures show that when the horizontal tail swings sinusoidally from its trimmed state, the forward flight velocity $\delta u^{+}$, fluctuating velocity $\delta w^{+}$, and pitch angular velocity $\delta q^{+}$all oscillate in a stable limit cycle. During this process, the trajectory of the vehicle in the longitudinal plane is as shown in Figure 7(d), and the FMAV enters an approximately elliptical periodic orbit through an arc trajectory. Figure $7(\mathrm{c})$ shows that when the tailed FMAV spirals in the longitudinal plane, the pitch attitude angle $\delta \theta^{+}$ begins to oscillate periodically about the longitudinal axis of the vehicle fuselage, along with the pitch angular velocity $\delta q^{+}$. Our results extend Lee's findings [23] and indicate that for the biplane FMAV, when the tail swings sinusoidally, the kinematic state variables also enter stable limit cycle oscillations. Furthermore, the elliptical flight trajectory in Figure 7 (d) shows that the sinusoidally swinging tail not only improves the passive stability of the aircraft, as found by Ravi et al. (2015) but also produces a spiraling flight mode in the longitudinal plane.

\section{Conclusion}

In this paper, we report studies of the disturbed stabilization in the longitudinal channel of a hovering tailed FMAV, as well as its flight control from hovering equilibrium. We began by modeling the longitudinal dynamics of a tailed FMAV and the way it is trimmed when hovering. Then, based on the small disturbance assumption, we linearized the longitudinal dynamic equations at the equilibrium point. We conducted research on the following three aspects of the hovering tailed FMAV:

(1) Stability analysis of the longitudinal dynamics of the tailed biplane FMAV: even though an FMAV can achieve a steady state by trimming with its tail, the vehicle is still dynamically unstable when there exist disturbances. Motion modal analysis shows that the tailed FMAV contains an oscillation divergence mode, a fast convergence mode, and a slow convergence mode in the longitudinal plane, which shows that the tailed FMAV has natural motion modes similar to those of tailless insects as described in [19]. However, because the mass and load distribution of the FMAV differs from that of insects, the dynamic diverging process of the FMAV is different from that of insects.

(2) In order to improve the stability of the tailed FMAV under disturbances, a state feedback controller was synthesized; it modulates the horizontal deviation angle of the tail. The bumblebee's recovering time from disturbance was chosen as the dynamic performance index, based on which the poles of the closed-loop system were configured. The results show that the state feedback controller can not only quickly suppress the disturbance to the hovering vehicle but also can reduce the oscillation in the response process.

(3) Based on the stability analysis of the longitudinal dynamics, we studied the flight control of the FMAV as it deviates from hovering. Both a step function and a sinusoidal function were selected as the control signals for the tail. 


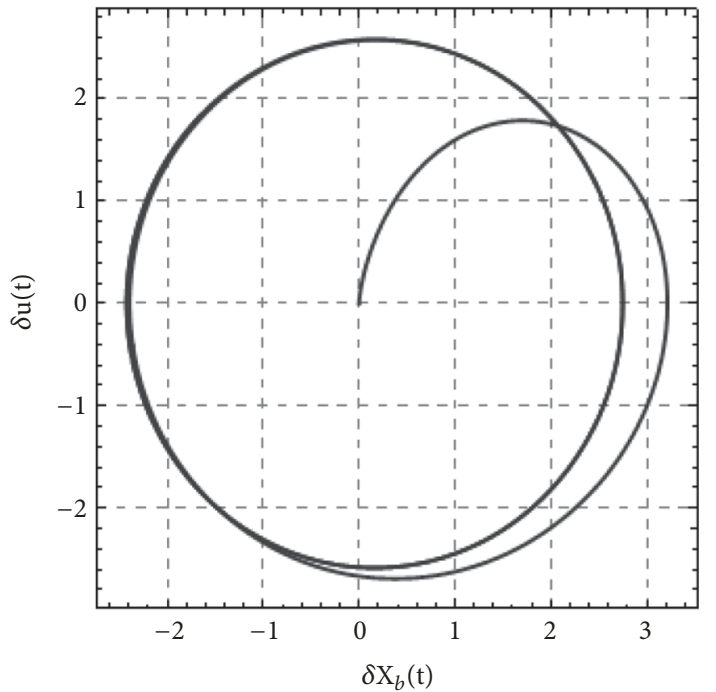

(a)

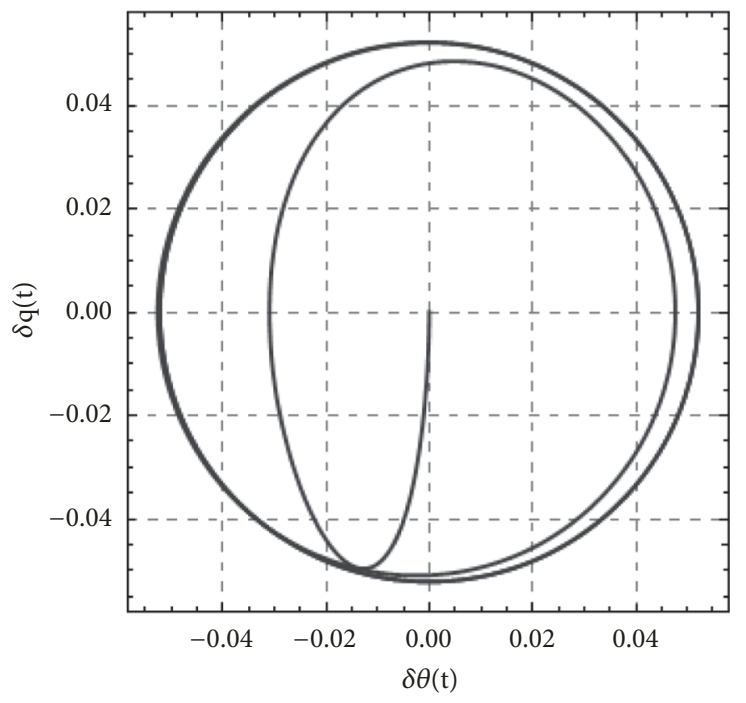

(c)

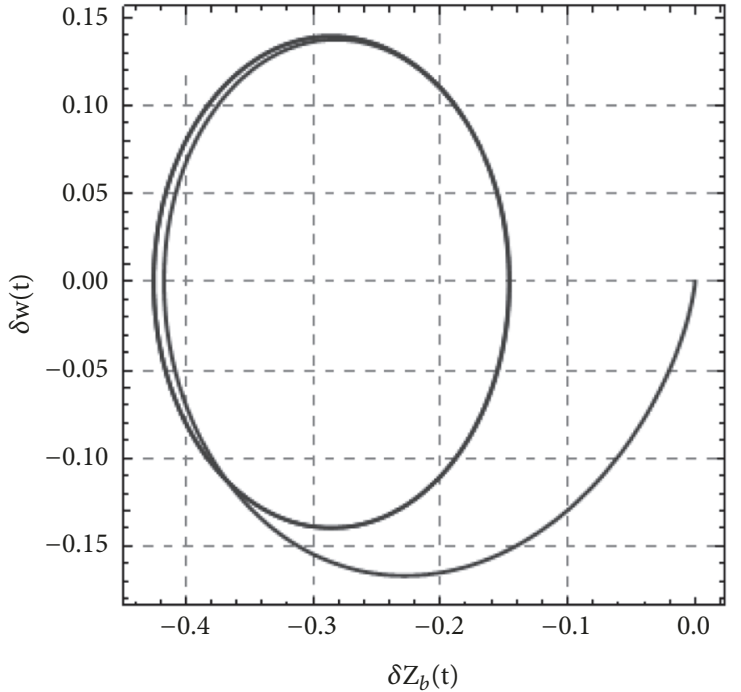

(b)

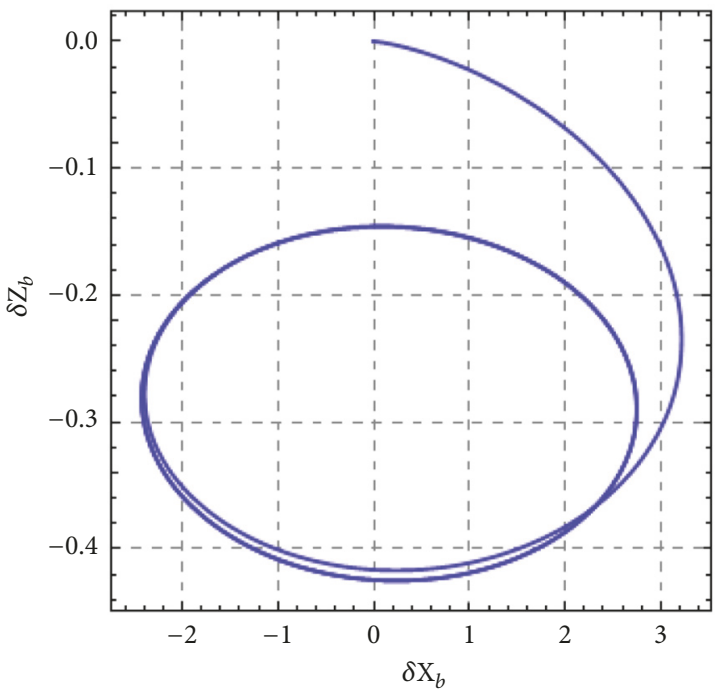

(d)

FIGURE 7: Phase diagram and flight trajectory of the FMAV with its tail vibrating sinusoidally.

The results show that when the tail deviates from its trim status as a step function, the vehicle starts to cruise from the hovering equilibrium, and both the forward flight velocity and fluctuating velocity are proportional to the magnitude of the step function. When the tail swings periodically, driven by a sinusoidal function, the kinematic state variables of the vehicle converge to a stable limit cycle oscillation (LCO). This result extends the finding of Lee et al. (2011) that the FMAV can spiral with its swinging tail in an elliptical periodic orbit in the longitudinal plane.

Since we included the aerodynamic effect of the tail as well as of the bionic wings, our model offers more precise analytical results than other quasisteady FMAV dynamic models. It should be pointed out that the longitudinal dynamic equation of the tailed FMAV, the attitude stabilization controller, and the flight control method from hovering equilibrium are all based on the small- disturbance assumption. Consequently, they are not suitable for understanding large-disturbance environments such as gusts. According to relevant research [20], the linearized longitudinal dynamic model is only applicable to the case where changes in the state variables of the FMAV are within 10\%; i.e., the wind field is stable or the vehicle starts to cruise at low speed from hovering equilibrium. If the FMAV is disturbed by strong gusts, nonlinear control methods should be considered. Also, when the vehicle enters a higher flight speed from hovering, it is necessary to reconsider the aerodynamic derivatives of the FMAV. We plan to work on both of these issues in the future. In this study, we proposed a set of control methods for the tailed FMAV for stable hovering, cruising at low speed, and spiraling in the longitudinal plane. We hope this work may contribute to the design of FMAVs to perform more complex missions, such as unmanned reconnaissance and search and rescue in tight spaces. 


\section{Data Availability}

The data used to support the findings of this study are available from the corresponding author upon request.

\section{Conflicts of Interest}

The authors declare no conflicts of interest.

\section{Acknowledgments}

This research was funded by National Defense Basic Scientific Research Program of China, grant number B1320133018.

\section{References}

[1] C. P. Ellington, "The aerodynamics of hovering insect flight. II. Morphological parameters," Philosophical Transactions of the Royal Society B: Biological Sciences, vol. 305, no. 1122, pp. 17-40, 1984.

[2] C. P. Ellington, "The aerodynamics of hovering insect flight. III. Kinematics," Philosophical Transactions of the Royal Society B: Biological Sciences, vol. 305, no. 1122, pp. 41-78, 1984.

[3] C. P. Ellington, "The aerodynamics of hovering insect flight. IV. Aeorodynamic mechanisms," Philosophical Transactions of the Royal Society B: Biological Sciences, vol. 305, no. 1122, pp. 79$113,1984$.

[4] B. W. Tobalske, "Biomechanics of bird flight," Journal of Experimental Biology, vol. 210, no. 18, pp. 3135-3146, 2007.

[5] F. T. Muijres, P. Henningsson, M. Stuiver, and A. Hedenström, "Aerodynamic flight performance in flap-gliding birds and bats," Journal of Theoretical Biology, vol. 306, pp. 120-128, 2012.

[6] E. de Margerie, J. B. Mouret, S. Doncieux, and J. Meyer, "Artificial evolution of the morphology and kinematics in a flapping-wing mini-UAV," Bioinspiration \& Biomimetics, vol. 2, no. 4, pp. 65-82, 2007.

[7] K. Y. Ma, P. Chirarattananon, S. B. Fuller, and R. J. Wood, "Controlled flight of a biologically inspired, insect-scale robot," Science, vol. 340, no. 6132, pp. 603-607, 2013.

[8] A. Ramezani, S. Chung, and S. Hutchinson, "A biomimetic robotic platform to study flight specializations of bats," Science Robotics, vol. 2, no. 3, Article ID eaal2505, 2017.

[9] M. Karásek, F. T. Muijres, C. De Wagter, B. D. Remes, and G. C. de Croon, "A tailless aerial robotic flapper reveals that flies use torque coupling in rapid banked turns," Science, vol. 361, no. 6407, pp. 1089-1094, 2018.

[10] H. V. Phan, T. Kang, and H. C. Park, "Design and stable flight of a $21 \mathrm{~g}$ insect-like tailless flapping wing micro air vehicle with angular rates feedback control," Bioinspiration \& Biomimetics, vol. 12, no. 3, Article ID 036006, 2017.

[11] S. Ravi, J. D. Crall, L. McNeilly, S. F. Gagliardi, A. A. Biewener, and S. A. Combes, "Hummingbird flight stability and control in freestream turbulent winds," Journal of Experimental Biology, vol. 218, no. 9, pp. 1444-1452, 2015.

[12] M. Sun, J. Wang, and Y. Xiong, "Dynamic flight stability of hovering insects," Acta Mechanica Sinica, vol. 23, no. 3, pp. 231246, 2007.

[13] G. C. H. E. de Croon, M. Perçin, B. D. W. Remes, R. Ruijsink, and C. De Wagter, The DelFly: Design, Aerodynamics, and Artificial Intelligence of a Flapping Wing Robot, Springer, Dordrecht, Netherlands, 2016.
[14] E. Ackerman, "This Robot Hummingbird Is Almost as Agile as the Real Thing: Purdue roboticists have built a bio-inspired micro air vehicle that flies much like a real hummingbird," https://spectrum.ieee.org/automaton/robotics/drones/robot-hummingbird-is-almost-as-agile-as-the-real-thing, 2019.

[15] S. Armanini, J. Caetano, C. de Visser, M. Pavel, G. de Croon, and M. Mulder, "Modelling wing wake and tail aerodynamics of a flapping-wing micro aerial vehicle," International Journal of Micro Air Vehicles, vol. 11, Article ID 175682931983367, 2019.

[16] S. S. Jadhav, K. B. Lua, and W. B. Tay, "Effect of clap-and-fling mechanism on force generation in flapping wing micro aerial vehicles," Bioinspiration \& Biomimetics, vol. 14, no. 3, Article ID 036006, 2019.

[17] J. V. Caetano, M. B. Weehuizen, C. C. de Visser, G. C. H. E. de Croon, and M. Mulder, "Rigid-body kinematics versus flapping kinematics of a flapping wing micro air vehicle," Journal of Guidance, Control, and Dynamics, vol. 38, no. 12, pp. 2257-2269, 2015.

[18] M. Karásek and A. Preumont, "Flapping flight stability in hover: a comparison of various aerodynamic models," International Journal of Micro Air Vehicles, vol. 4, no. 3, pp. 203-226, 2012.

[19] M. Sun, "Dynamic flight stability of a hovering bumblebee," Journal of Experimental Biology, vol. 208, no. 3, pp. 447-459, 2005.

[20] J. Wu and M. Sun, "Control for going from hovering to small speed flight of a model insect," Acta Mechanica Sinica, vol. 25, no. 3, pp. 295-302, 2009.

[21] S. Ravi, D. Kolomenskiy, T. Engels et al., "Bumblebees minimize control challenges by combining active and passive modes in unsteady winds," Scientific Reports, vol. 6, no. 1, Article ID 35043, 2016.

[22] T. Beatus, J. M. Guckenheimer, and I. Cohen, "Controlling roll perturbations in fruit flies," Journal of the Royal Society Interface, vol. 12, no. 105, Article ID 20150075, 2015.

[23] J. Lee, J. Kim, J. Han, and C. P. Ellington, "Periodic tail motion linked to wing motion affects the longitudinal stability of ornithopter flight," Journal of Bionic Engineering, vol. 9, no. 1, pp. 18-28, 2012. 


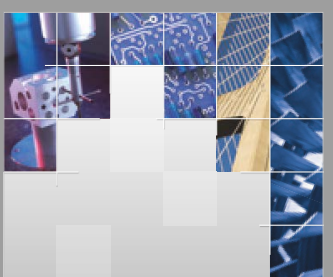

\section{Enfincering}
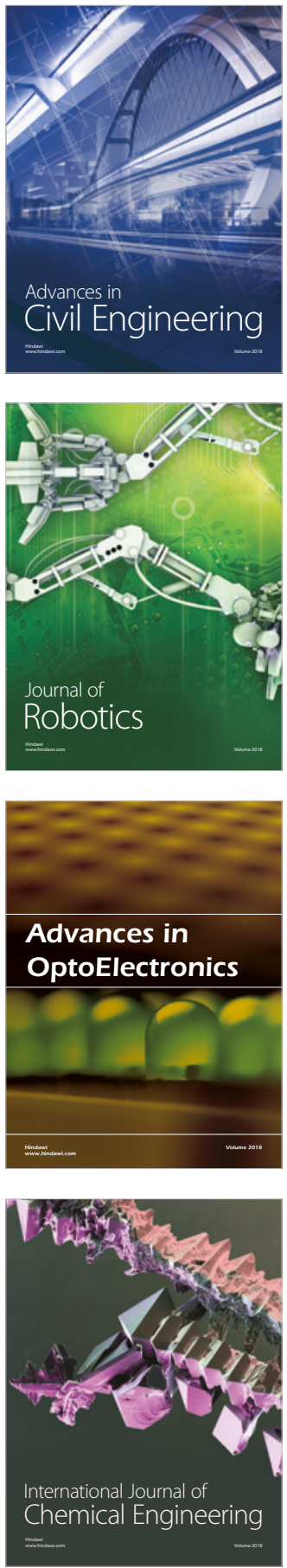

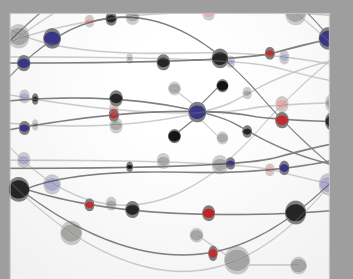

\section{Rotating \\ Machinery}

The Scientific World Journal

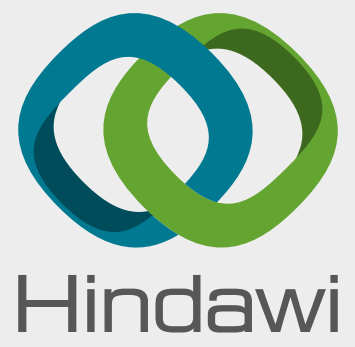

Submit your manuscripts at

www.hindawi.com
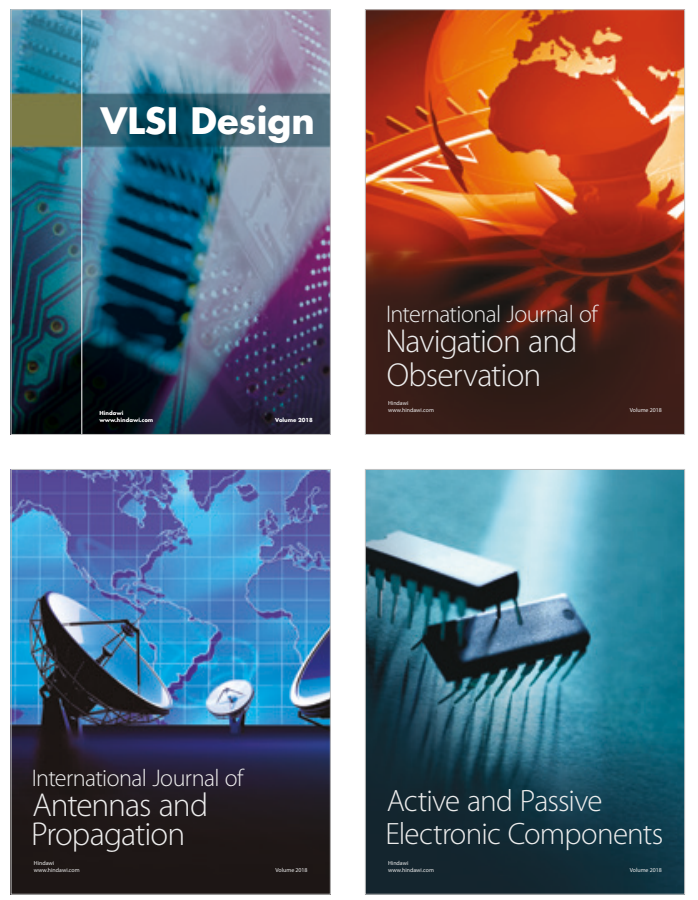
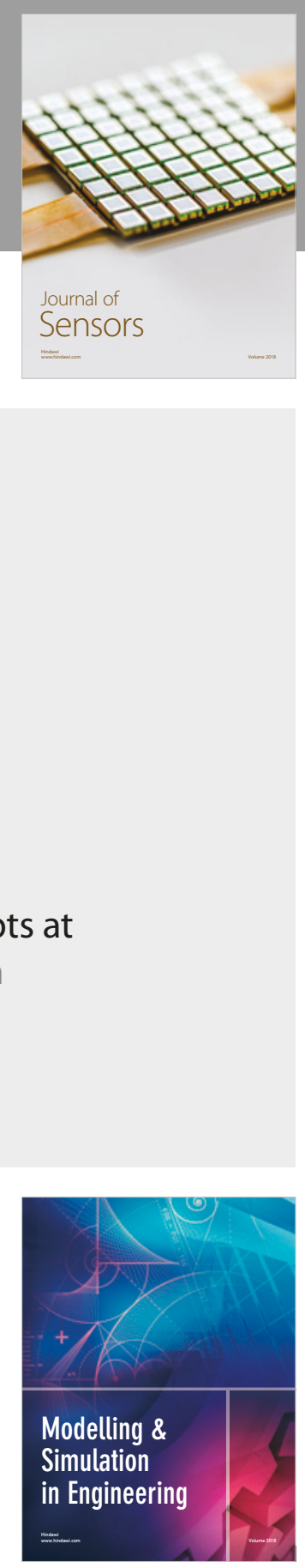

\section{Advances \\ Multimedia}
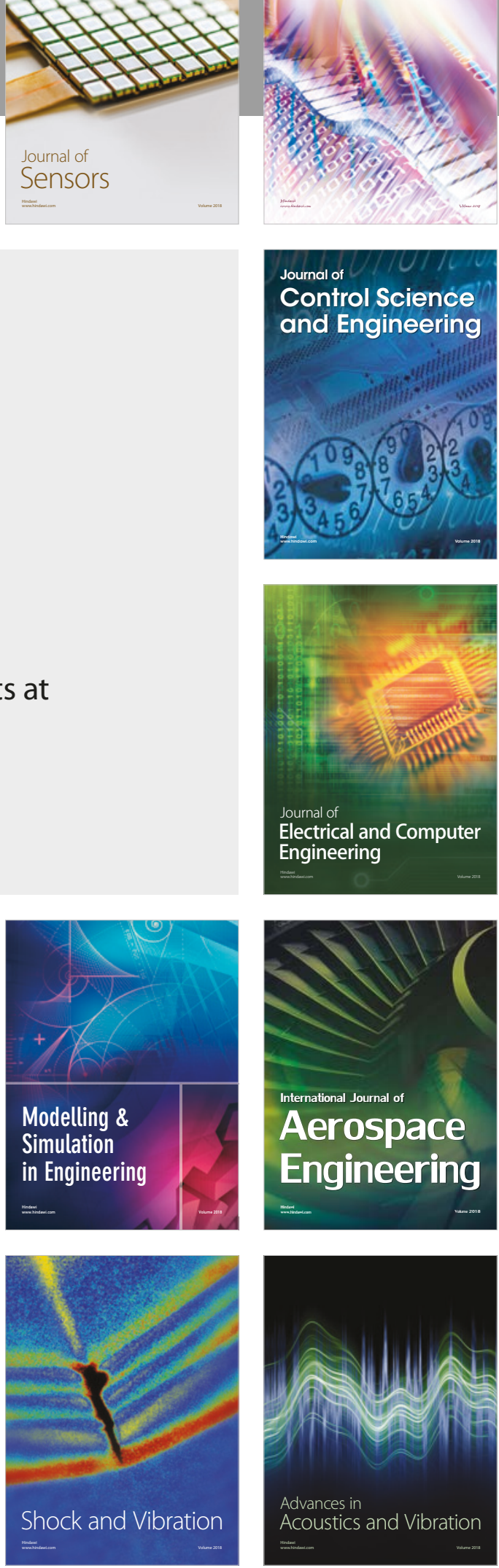